\title{
Letter on "Pre-hospital transthoracic echocardiography for early identification of non-ST-elevation myocardial infarction in patients with acute coronary syndrome"
}

\author{
Guido Tavazzi ${ }^{1,2^{*}}$ (D) Aleksandar N Neskovic ${ }^{3,4}$, Bogdan A Popescu ${ }^{5}$ and Gabriele Via ${ }^{6}$ \\ See related research by Bergmann et al., https://ccforum.biomedcentral.com/articles/10.1186/s13054-017-1929-1
}

Keywords: Focused cardiac ultrasound, Point of care ultrasound, Echocardiography, Myocardial infarction, Non ST-elevation myocardial infarction

We read with interest the manuscript by Bergmann et al. [1] but believe it is fraught by several conceptual and methodological flaws, the main ones being:

- The authors used interchangeably the terms "transthoracic echocardiography", "focus echocardiography", and "focus cardiac ultrasound"(FoCUS) without the clear distinction required by the potential major clinical implications [2]; the screening for regional wall motion abnormalities (RWMA) is in fact clearly considered by international consensus beyond the scope of the limited training and application that FoCUS entails $[3,4]$, and the level of echocardiographic education/ competence of emergency physicians was not detailed.

- The authors state: "A diagnosis of NSTEMI was based on the combination of ACS symptoms, lack of ST-segment elevation, and RWMA. Myocardial infarction was excluded in the absence of the latter." Non-transmural infarctions compromising a small amount of necrotic myocardium may not be detectable on 2D-echo. It has been shown that RWMA detectable by echocardiography occur if

\footnotetext{
* Correspondence: gtavazzi@yahoo.it

1Department of Clinical, Surgical, Diagnostic and Paediatric Sciences,

Anaesthesia, Intensive Care and Pain Therapy Unit, University of Pavia, Pavia, Italy

Emergency Department, Anaesthesia and Intensive Care Unit, Fondazione

IRCCS Policlinico S. Matteo, Pavia, Italy

Full list of author information is available at the end of the article
}

resting coronary flow is reduced by $>50 \%$, if $>20 \%$ of myocardial thickness is jeopardized by actual ischemia/necrosis, or if at least $1-6 \%$ of the left ventricle mass is involved [5].

- Previous myocardial infarction is indicated as an exclusion criterion. But a screening for myocardial scars or signs of pre-existing left ventricle disease was omitted from the exam, and a subsequent re-reading of the images by a blinded expert was omitted too, which may have led to potential false positives in non-ST-segment myocardial infarction (NSTEMI) diagnosis.

It remains obscure how the authors can conclude that "No evidence of myocardial infarction was found in any patient with NSTE-ACS without RWMA in the pre-hospital TTE" and later state that NSTEMI was conclusively diagnosed in two patients without RWMA [1]. We agree, as recommendations do [2], that pre-hospital ultrasound has the potential to lead to earlier diagnosis and faster treatment in acute cardiac patients. But, based on questionable methodology and unclear data, this study conveys the equivocal message that FoCUS has sufficient diagnostic accuracy for NSTEMI.

(c) The Author(s). 2018 Open Access This article is distributed under the terms of the Creative Commons Attribution 4.0 International License (http://creativecommons.org/licenses/by/4.0/), which permits unrestricted use, distribution, and reproduction in any medium, provided you give appropriate credit to the original author(s) and the source, provide a link to the Creative Commons license, and indicate if changes were made. The Creative Commons Public Domain Dedication waiver (http://creativecommons.org/publicdomain/zero/1.0/) applies to the data made available in this article, unless otherwise stated. 


\section{Abbreviations}

ACS: Acute coronary syndrome; FoCUS: Focus cardiac ultrasound; MI: Myocardial infarction; NSTE-ACS: Non-ST-elevation acute coronary syndrome; NSTEMI: Non-ST-segment myocardial infarction; RWMA: Regional wall motion abnormality; TTE: Transthoracic echocardiography

\section{Availability of data and materials}

Data sharing is not applicable to this article as no datasets were generated or analyzed during the current study.

\section{Authors' contributions}

All the authors contributed equally. All authors read and approved the final manuscript.

Ethics approval and consent to participate

Not applicable.

\section{Consent for publication}

Not applicable.

\section{Competing interests}

The authors declare that they have no competing interests.

\section{Publisher's Note}

Springer Nature remains neutral with regard to jurisdictional claims in published maps and institutional affiliations.

\section{Author details}

${ }^{1}$ Department of Clinical, Surgical, Diagnostic and Paediatric Sciences, Anaesthesia, Intensive Care and Pain Therapy Unit, University of Pavia, Pavia, Italy. ${ }^{2}$ Emergency Department, Anaesthesia and Intensive Care Unit, Fondazione IRCCS Policlinico S. Matteo, Pavia, Italy. ${ }^{3}$ Department of Cardiology, Clinical Hospital Center Zemun, Belgrade, Serbia. ${ }^{4}$ Faculty of Medicine, University of Belgrade, Belgrade, Serbia. ${ }^{5}$ University of Medicine and Pharmacy "Carol Davila" - Euroecolab Emergency Institute of Cardiovascular Diseases "Prof. Dr. C. C. Iliescu", Bucharest, Romania. ${ }^{6}$ Cardiac Anesthesia and Intensive Care, Cardiocentro Ticino, Lugano, Switzerland.

Received: 19 July 2018 Accepted: 24 September 2018

Published online: 21 November 2018

\section{References}

1. Bergmann I, Buttner B, Teut E, Jacobshagen C, Hinz J, Quintel M, Mansur A, Roessler M. Pre-hospital transthoracic echocardiography for early identification of non-ST-elevation myocardial infarction in patients with acute coronary syndrome. Crit Care. 2018;22(1):29.

2. Neskovic AN, Hagendorff A, Lancellotti P, Guarracino F, Varga A, Cosyns B, Flachskampf FA, Popescu BA, Gargani L, Zamorano JL. Emergency echocardiography: the European association of cardiovascular imaging recommendations. Eur Heart J Cardiovasc Imaging. 2013;14(1):1-11.

3. Via G, Hussain A, Wells M, Reardon R, ElBarbary M, Noble VE, Tsung JW, Neskovic AN, Price S, Oren-Grinberg A, et al. International evidence-based recommendations for focused cardiac ultrasound. J Am Soc Echocardiogr. 2014;27(7):683 e681-683 e633.

4. Labovitz AJ, Noble VE, Bierig M, Goldstein SA, Jones R, Kort S, Porter TR, Spencer KT, Tayal VS, Wei K. Focused cardiac ultrasound in the emergent setting: a consensus statement of the American Society of Echocardiography and American College of Emergency Physicians. J Am Soc Echocardiogr. 2010;23(12):1225-30.

5. Roffi M, Patrono C, Collet JP, Mueller C, Valgimigli M, Andreotti F, Bax JJ, Borger MA, Brotons C, Chew DP, et al. 2015 ESC Guidelines for the management of acute coronary syndromes in patients presenting without persistent ST-segment elevation: Task Force for the Management of Acute Coronary Syndromes in Patients Presenting without Persistent ST-Segment Elevation of the European Society of Cardiology (ESC). Eur Heart J. 2016;37(3):267-315 